\title{
Research on Loss-averse Newsboy Problem Based on Uncertain Capacity
}

\author{
Kangkang Ge
}

School of Economics \& Management, Jiangsu University of Science and Technology, Zhenjiang, China

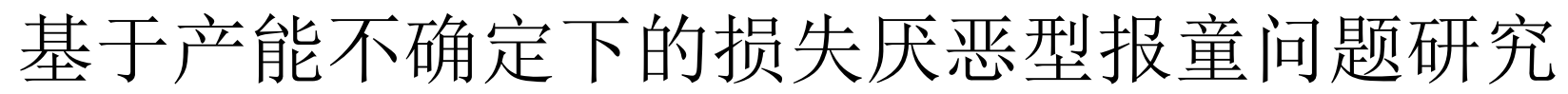

\author{
葛康康 \\ 江苏科技大学 经济管理学院, 江苏 镇江, 中国
}

\begin{abstract}
In the past, the research on the newsboy issue was mainly based on the basic assumption that the market demand was uncertain, while ignoring the possible impact of uncertain supplier capacity. In this paper, a loss-averse newsboy model considering the cost of out-of-stock punishment and uncertainty of supplier's capacity is constructed for this problem. Based on the assumption that stochastic demand is evenly distributed, the optimal ordering strategy for loss-averse retailers with uncertain production capacity is studied. The research in this paper shows that the optimal order quantity of the retailer is positively correlated with the residual value of the commodity unit and negatively correlated with the loss aversion coefficient. In addition, it has been found that, under certain conditions, the optimal order quantity may increase or decrease with the increase in the ordering cost, sales price, or out-of-stock penalty cost of the product.
\end{abstract}

Keywords-random capacity; loss aversion; uniform distribution; newsboy model

摘要一以往对于报童问题的研究主要基于市场需求不确定这一 基本假设, 而忽略了供应商产能不确定可能带来的影响。本文针 对该问题构建了考虑缺货惩罚成本且供应商产能不确定下的损 失厌恶型报童模型, 基于随机需求服从均匀分布的假设, 研究 产能不确定下损失厌恶型零售商的最优订购策略。文中的研究 表明零售商的最优订购量与商品单位残值正相关、与损失厌恶 系数负相关。此外发现, 在一定条件下, 随着商品订购成本、 销售价格或缺货惩罚成本的增加, 最优订购量可能增加, 也可 能减小。

关键词一随机产能; 损失厌恶; 均匀分布; 报童模型

$$
\text { I. 引言 }
$$

作为随机库存理论的基本模型之一，报童问题长期以 来被广泛地应用于产能规划、收益管理、供应链管理等研 究之中。传统的报童模型问题是一种单期随机库存模型。 后来的研究发现, 决策者的实际订购行为与理论上的最优
订购量总是存在一些偏差。Fisher 和 Raman[1]发现时装零 售商的订购量会低于期望理论模型下的最优订购量。 Schweitzer 与 Cachon[2]通过实验研究发现零售商的实际订 货行为系统地偏离最优订货量, 认为决策者在风险、损 失、浪费、缺货等八个方面的倾向会对其订购量产生影 响。Wang 和 Webster[3]构建了损失厌恶型报童模型研究损 失厌恶态度对决策者决策行为的影响。研究发现当考虑缺 货惩罚成本时，损失厌恶型报童的订购量可能会大于风险 中性报童。其最优订购量可能会随订购成本的增加而增 加、随销售价格的上升而减小。Xu 等[4]将损失厌恶的分 段线性效用函数引入条件风险价值 (Conditional Value-At-Risk, CVaR)中, 发现在该目标最大化条件下得 到的最优订购量随置信水平的上升而减小，并且比期望效 用下的最优订购量小。Wang[5]研究了损失厌恶型报童的 博弯问题。结果表明如果供应商对零售商的订购量按比例 将商品分配给零售商, 则存在唯一的纳什均衡解。在报童 问题中, 一般假设消费者的需求为随机的, 这种需求是库 存系统随机性的主要来源。然而, 消费者需求并不是系统 随机性的唯一来源。实际上, 近年来关于供应商的供应不 确定性研究也开始受到关注。影响供应商无法满足零售商 订货量的因素较多, 比如机器故障导致的停工、运输过程 中商品的损坏或变质、工人罢工、自然灾害等等都会对供 应商的按量供应产生不利影响。Karlin[6]最早研究生产不 确定性问题, Yano 和 Lee[7]对生产不确定性问题给出了详 细的综述。Keren[8]研究了确定性需求但供应为随机时的 单期库存问题，分析了加式与乘式随机生产情形，得出随 机生产服从均匀分布下的最优解, 发现零售商的最优订购 量可能大于确定性供应时的最优订购量。 $\mathrm{Li}$ 等 [9]在 Keren[8]研究的基础上, 考虑了更为一般的随机生产分布 形式。他们指出, 订购更多的商品并非总是最优策略, 并 给出了策略最优的条件。此外发现随机生产下供应链利润 会减少, 但供应商却可能会因此获益。 
上述关于损失厌恶型报童问题的研究，要么单方面基 于随机需求的假设, 要么同时考虑市场需求和供应商供应 均为不确定情形, 鲜有研究考虑缺货惩罚成本且同时考虑 需求和供应均为随机情形下的损失厌恶型报童问题。为 此，本文构建考虑缺货惩罚成本且供应商产能不确定下的 损失厌恶型报童模型, 研究了损失厌恶型零售商的最优订 购策略以及系统参数对订购量的影响。

\section{II. 模型描述}

假设零售商同时面临随机需求和随机供应两种情形, 并 且这两个随机变量相互独立。不失一般性, 我们令 $F(0)=G(0)=0$, 其中 $F(\cdot)$ 和 $G(\cdot)$ 分别表示随机需求和随机 产能的累积分布函数。本文模型中所用到的其他变量及其 符号如下所示: 单位商品订购成本 $C$ 、单位商品销售价 格 $p$ 、剩余商品单位残值 $v$ 、单位缺货惩罚成本 $s$ 、零售 商的订购量 $q$ 、市场随机需求变量 $X$ 、供应商随机产能

$Y$ 、随机需求概率密度函数 $f(x)$ 、随机产能概率密度函 数 $g(y)$ 、利润函数 $\pi(\cdot)$ 、效用函数 $U(\cdot)$ 、损失厌恶系 数 $\lambda(>1)$ 。 $\lambda$ 越大, 损失厌恶程度越大。零售商的利润函 数可表示为:

$$
\begin{aligned}
\pi(q, x, y)= & (p+s-c) \min (y, q)-(p+s-v)(\min (y, q)-x)^{+}-s x \\
& =\left\{\begin{array}{cc}
-(c-v) y+(p-v) x, & x<y \leq q \\
(p+s-c) y-s x, & y \leq q, y \leq x \\
-(c-v) q+(p-v) x, & x<q<y \\
(p+s-c) q-s x, & q<y, q \leq x
\end{array}\right.
\end{aligned}
$$

关于损失厌恶型零售商的效用描述，我们采用文献[3] 中所使用的分段线性效用函数形式, 即:

$$
U(\pi)=\left\{\begin{array}{cc}
\pi, & \pi \geq 0 \\
\lambda \pi, & \pi<0
\end{array}\right.
$$

上式表示当零售商销售利润大于零时, 效用等于其所获 得的利润值; 当销售利润为负值 (亏损)时, 对于零售商而言, 这种负效用被放大为 $\lambda \pi$, 对损失表现出厌恶的态度。综合 (1)和(2)可得损失厌恶型零售商的效用函数:

$$
U[\pi(q, x, y)]=\left\{\begin{array}{cc}
-(c-v) y+(p-v) x, & \frac{c-v}{p-v} y \leq x<y \leq q \\
\lambda[-(c-v) y+(p-v) x], & x<\frac{c-v}{p-v} y, y \leq q \\
(p+s-c) y-s x, & y \leq q, \frac{s}{p+s-c} x \leq y \leq x \\
\lambda[(p+s-c) y-s x], & y \leq q, 0<y<\frac{s}{p+s-c} x \\
-(c-v) q+(p-v) x, & \frac{c-v}{p-v} q \leq x<q<y \\
\lambda[-(c-v) q+(p-v) x], & x<\frac{c-v}{p-v} q, q<y \\
(p+s-c) q-s x, & q<y, q \leq x \leq \frac{p+s-c}{s} q \\
\lambda[(p+s-c) q-s x], & q<y, x>\frac{p+s-c}{s} q
\end{array}\right.
$$

由(3),损失厌恶型零售商的期望效用函数可表示为:

$$
\begin{aligned}
& E[U(\pi(q, x, y))] \\
& =\int_{0}^{q} \int_{\frac{c-v}{p-v} y}^{y}[-(c-v) y+(p-v) x] d F(x) d G(y)+\int_{0}^{q} \int_{0}^{\frac{c-v}{p-v} y} \lambda[-(c-v) y+(p-v) x] d F(x) d G(y) \\
& +\int_{0}^{q} \int_{y}^{\frac{p+s-c}{s} y}[(p+s-c) y-s x] d F(x) d G(y)+\int_{0}^{q} \int_{\frac{p+s-c}{s} y}^{\infty} \lambda[(p+s-c) y-s x] d F(x) d G(y) \\
& +\int_{q}^{\infty} \int_{\frac{c-v}{p-v} q}^{q}[-(c-v) q+(p-v) x] d F(x) d G(y)+\int_{q}^{\infty} \int_{0}^{\frac{c-v}{p-v} q} \lambda[-(c-v) q+(p-v) x] d F(x) d G(y) \\
& +\int_{q}^{\infty} \int_{q}^{\frac{p+s-c}{s} q}[(p+s-c) q-s x] d F(x) d G(y)+\int_{q}^{\infty} \int_{\frac{p+s-c}{s} q}^{\infty} \lambda[(p+s-c) q-s x] d F(x) d G(y) \\
& =E[\pi(q, x, y)]+ \\
& (\lambda-1)\left\{\begin{array}{l}
\int_{0}^{q} \int_{0}^{\frac{c-v}{p-v} y}[-(c-v) y+(p-v) x] d F(x) d G(y)+\int_{0}^{q} \int_{\frac{p+s-c}{s} y}^{\infty}[(p+s-c) y-s x] d F(x) d G(y) \\
+\int_{q}^{\infty} \int_{0}^{\frac{c-v}{p-v} q}[-(c-v) q+(p-v) x] d F(x) d G(y)+\int_{q}^{\infty} \int_{\frac{p+s-c}{s} q}^{\infty}[(p+s-c) q-s x] d F(x) d G(y)
\end{array}\right\}
\end{aligned}
$$


从上式结果中可以看出, 损失厌恶型零售商的期望效用 函数为风险中性零售商的期望效用加上期望损失乘以 $(\lambda-1)$ 。当 $\lambda=1$ 时, 上式结果中第二次项去掉, 即变为了 风险中性情形。

命题 1 期望效用函数 $E[U(\pi(q, x, y))]$ 为订购量 $q$ 的 凹函数, 且存在唯一最优订购量 $q_{\lambda}^{*}$ 满足下面一阶条件:

$$
(\lambda-1)\left[(p+s-c) F\left(\frac{p+s-c}{s} q_{\lambda}^{*}\right)+(c-v) F\left(\frac{c-v}{p-v} q_{\lambda}^{*}\right)\right]+(p+s-v) F\left(q_{\lambda}^{*}\right)=\lambda(p+s-c)
$$

证明 见附录。当 $s \rightarrow 0^{+}$时, 命题 1 中的一阶条件就变 为了文献[12]不考虑惩罚成本的情形, 即:

$(\lambda-1)(c-v) F\left(\frac{c-v}{p-v} q_{\lambda}^{*}\right)+(p-v) F\left(q_{\lambda}^{*}\right)=p-c$ 。可见考虑缺 货惩罚成本时比不考虑缺货惩罚成本情况复杂得多。另外, 当 $\lambda=1$ 时, 相应变为了风险中性时的情形, 即 $q_{1}^{*}=F^{-1}\left(\frac{p+s-c}{p+s-v}\right)$ 。可见当供应商的产能不确定时, 对风险 中性零售商的最优订购量并没有产生影响。这个结果与我 们的直觉也是相符合的, 如果风险中性零售商的订购量大 于或小于最优订购量 $q_{\lambda}^{*}$, 那么就无法实现期望利润最大化。

\section{III. 系统参数分析}

以下部分假设市场随机需求服从均匀分布进行分析。

命题 2 当随机需求 $X \sim U[a, b]$ 时, 对于任意 $\lambda>1$, 损 失厌恶零售商的最优订购量总是小于风险中性零售商。

证明 见附录。文献[3]表明, 当市场需求为系统随机性的 唯一来源时, 带有缺货惩罚成本的损失厌恶型零售商的最 优订购量可能大于风险中性零售商。与之不同的是, 本文 同时考虑零售商面临市场不确定和供应商供应不确定情况 下, 且随机需求服从均匀分布时, 损失厌恶零售商的最优 订购量总是小于风险中性零售商, 可见供应商的产能不确 定会影响零售商的决策行为。

推论 1 当随机需求 $X \sim U[a, b]$ 时, 损失厌恶零售商的最 优订购量 $q_{\lambda}^{*}$ 随损失厌恶系数 $\lambda$ 的增加而减小。

证明见附录。

推论 2 随机需求 $X \sim U[a, b]$, 当

(1) $b>\frac{\lambda(c-v) a}{(\lambda-1)\left[\frac{(p+s-c)^{2}}{s}+\frac{(c-v)^{2}}{(p-v)}\right]+(p+s-v)-\lambda(p+s-c)}$, $0<s<\frac{1+\sqrt{5}}{2}(p-c)$,
或(2)

$$
<\frac{\lambda(c-v) a}{(\lambda-1)\left[\frac{(p+s-c)^{2}}{s}+\frac{(c-v)^{2}}{(p-v)}\right]+(p+s-v)-\lambda(p+s-c)},
$$

$0<s<\frac{1+\sqrt{5}}{2}(p-c), \lambda>\frac{1+\frac{(p+s-c) \cdot L(s)}{s^{3}}}{\frac{b}{q_{\lambda}^{*}}+\frac{(p+s-c) \cdot L(s)}{s^{3}}}$ 时, 最优订购量 $q_{\lambda}^{*}$

随缺货惩罚成本 $s$ 的增加而增加; 当

$b<\frac{\lambda(c-v) a}{(\lambda-1)\left[\frac{(p+s-c)^{2}}{s}+\frac{(c-v)^{2}}{(p-v)}\right]+(p+s-v)-\lambda(p+s-c)}$

$0<s<\frac{1+\sqrt{5}}{2}(p-c), \quad 1<\lambda<\frac{1+\frac{(p+s-c) \cdot L(s)}{s^{3}}}{\frac{b}{q_{2}^{*}}+\frac{(p+s-c) \cdot L(s)}{s^{3}}}$ 时, 最优订购量

随缺货惩罚成本的增加而减小。其中 $L(w)=-s^{2}+(p-c) s+(p-c)^{2}$ 。

证明见附录。推论 2 给出了随着缺货惩罚成本的增加, 零售商最优订购量随之增大或减小的充分条件。一般情况 下, 随着缺货惩罚成本的增加, 零售商会更加重视避免缺 货情况的发生, 往往会订购更多商品以避免缺货导致的损 失。推论 2 不同于我们的常识, 是一个反直觉的结论, 这 是由于产能不确定对损失厌恶型报童的订购决策带来的影 响。

推论 3 当随机需求 $X \sim U[a, b]$ 时, 损失厌恶型零售商的 最优订购量 $q_{\lambda}^{*}$ 随单位残值 $v$ 的增加而增大。

证明见附录。推论 3 表明随着剩余商品单位残值的增 加, 决策者的订购量随之增大。当单位残值增加时, 零售 商因剩货带来的损失减小, 可以想象, 当残值增大到订购 成本时, 零售商将不会因剩货产生损失。因此, 该推论与 我们的直觉是一致的。

$$
\begin{aligned}
& \text { 推 论 } 4 \quad X \sim U[a, b] \text {, } \\
& b \leq \frac{\lambda(c-v) a}{(\lambda-1)\left[\frac{(p+s-c)^{2}}{s}+\frac{(c-v)^{2}}{(p-v)}\right]+(p+s-v)-\lambda(p+s-c)} \text { 时, 最优 }
\end{aligned}
$$

订购量 $q_{\lambda}^{*}$ 随销售价格 $p$ 的增大而减小; 当 $1<\lambda<\frac{\frac{O(s)}{s^{2}(p-v)^{3}}-1}{\frac{O(s)}{s^{2}(p-v)^{3}}-\frac{b}{q_{\lambda}^{*}}}$

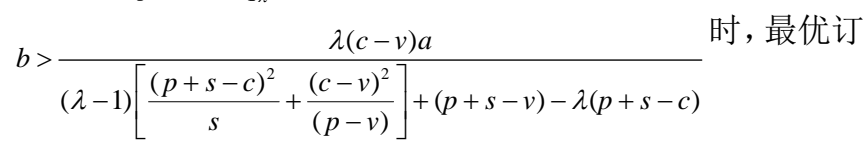

购量 $q_{\lambda}^{*}$ 随销售价格 $p$ 的增大而增大。其中 $O(s)=\left[2(p-v)^{3}-(c-v)^{3}\right] s^{2}+3(p-v)^{3}(p-c) s+(p-v)^{3}(p-c)^{2}$ 。

证明见附录。

推论 5 随机需求 $X \sim U[a, b]$, 当 $(p-v)(p-c)-(c-v)^{2}>0$ 且 $\lambda>1+\frac{(p+s-v) s^{2}(p-v)^{2}}{(p+s-c) T(s)-s(p-v)\left[(p-v)(p+s-c)^{2}+(c-v)^{2} s\right]}$ 时, 
最优订购量 $q_{\lambda}^{*}$ 随订购成本 $c$ 的增大而增加; 当 (1) $(p-v)(p-c)-(c-v)^{2}>0$

$$
1<\lambda<1+\frac{(p+s-v) s^{2}(p-v)^{2}}{(p+s-c) T(s)-s(p-v)\left[(p-v)(p+s-c)^{2}+(c-v)^{2} s\right]}
$$

$(p-v)(p-c)-(c-v)^{2}<0$ 时, 最优订购量 $q_{\lambda}^{*}$ 随订购成本 $c$ 的增大而减小其中 $T(s)=\left[2(p-v)^{2}-(c-v)(p+c-2 v)\right] s^{2}+3(p-v)^{2}(p-c) s+(p-v)^{2}(p-c)^{2}$ 。

证明见附录。

推论 5 给出了零售商最优订购量随订购成本的增加而增 大或减小的充分条件, 类似于推论 2 , 推论 5 也是一种反直 觉的结论。

\section{IV. 结束语}

本文在已有文献的基础上, 分析考虑缺货惩罚成本情形 时供应商产能不确定下的损失庈恶型报童模型, 研究了损 失厌恶型零售商的最优订购策略以及系统参数对零售商订 购量的影响。研究表明, 供应商产能不确定对风险中性零 售商的订购策略没有任何影响。在假设市场需求服从均匀 分布的条件下, 我们得出了零售商的最优订购量与商品单 位残值正相关、与损失庆恶系数负相关的结论。此外发现, 随着商品订购成本、销售价格或缺货惩罚成本的增加, 最 优订购量可能增加, 也可能减小。

\section{REFERENCE}

[1] Fisher M, Raman A. reducing the cost of demand uncertainty through accurate response to early sales[J]. Operations Research, 1996, 44(1):87-99.

[2] Schweitzer M E, Cachon G P. Decision bias in the newsvendor problem with a known demand distribution: Experimental evidence [J]. Management Science, 2000, 46(3):404-420.

[3] Charles X. Wang, Scott Webster. The loss-averse newsvendor problem[J]. Omega, 2009, 37(1):93-105.

[4] Xu X, Wang H, Dang C, et al. The loss-averse newsvendor model with backordering[J]. International Journal of Production Economics, 2017, 188:1-10.

[5] Wang C X. The loss-averse newsvendor game[J]. International Journal of Production Economics, 2010, 124(2):448-452.

[6] Arrow K J, Karlin S, Scarf H. Studies in the mathematical theory of inventory and production [M]. STANFORD UNIV. PR, 1958.

[7] Yano C A, Lee H L. Lot sizing with random yields: A review[J]. Operations Research, 1995, 43(2):311-334.

[8] Keren B. The single-period inventory problem: extension to random yield from the perspective of the supply chain [J]. Omega, 2009, 37(4):801-810.

[9] Li X, Li Y, Cai X. A note on the random yield from the perspective of the supply chain[J]. Omega, 2012, 40(5):601-610. 